tions of 50,000. A resolution of 100,000 was enough for Gray to find an asymmetry in the $6,253-\AA$ iron absorption line, similar to that caused by convection in other stars, with implied relative velocity of $\pm 45 \mathrm{~m} \mathrm{~s}^{-1}$. That is close to the velocities measured by Mayor and Queloz, and it has the same 4.23-day period. Further, the ratio of the central depths of two lines that are normally sensitive to temperature fluctuations varies with the same period. The data extend over many years and are consistent over hundreds of velocity cycles. These results are convincing enough to give pause, but should we abandon the planetary interpretation altogether?

Gray's result raises the puzzle: what possible variability of 51 Pegasi could generate a perfect sine-wave in its radial velocities? A mechanism connected with rotation might do that naturally, but there is indirect evidence $^{5}$ that the star's rotation period is about a month, far too long to cause the four-day variability. Perhaps any higherorder effects have been smoothed out by the low spectral resolution used in the Doppler detection or in the algorithm used to measure the line-shifts. Radial oscillations of the surface, on the other hand, would cause brightness fluctuations greater than those seen; and acoustic non-radial oscillations such as those on the Sun would have too short a period, and are unlikely to have remained coherent for hundreds of cycles.

Still, there has been ample evidence for some time that old solar-type stars - those that have evolved beyond the stable 'main sequence' - have a stable, low-amplitude velocity variability, with periods from days to years, which exactly mimics the signature of planetary reflex motion ${ }^{6}$. Gray points out that the absolute brightness of 51 Pegasi indicates that it is somewhat evolved, towards the sub-giant stage. Only by examining the spectral lines at sufficient spectral resolution can a 'Doppler map' be constructed of the star's surface using the star's rotation. Until such data are available, perhaps some time in the next year, we just can't be sure of the exact nature of any intrinsic variability.

So independent follow-up observations at even higher spectral resolution of 51 Pegasi and the other 3-14-day period candidates, $v$ Andromedae, 55 Cancri and $\tau$ Bootis, are essential. One startling outcome, if some of the short-period candidates have to be withdrawn, is that Jupiter-mass planets will become as rare as brown dwarfs, only two of which have actually been seen.

Gordon Walker is in the Physics and Astronomy

Department, University of British Columbia,

Vancouver, British Columbia V6T 1Z4, Canada.

1. Mayor, M. \& Qucloz, D. Nature 378, 355-359 (1995).

2. Marcy, G. W. et al. Astrophys. J. (submitted).

3. Lin, D. C. N., Bodenheimer, P. \& Richardson, D. C. Nature 380,

606-607(1996)

4. Gray, D. F. Nature 385, 795-796 (1997).

5. Baliunas, S. L. et al. Astrophys. J. 474, LI19-I.122 (1997)

6. Walker, G. A. FI. et al. Astrophys. J. 343, L21-24 (1989).
Conservation biology Case studies of extinction

Paul H. Harvey and Robert M. May

T he distributions of species are changing at unprecedented rates. Some are becoming extinct while others are expanding their ranges. To identify the general causes of these changes, as opposed to the particulars of individual cases, we need large data sets covering diverse areas.

Case $^{1}$ and Dobson et al. ${ }^{2}$, writing in Biological Conservation and Science respectively, have now produced and analysed examples of such data sets. For island birds, for instance, they reveal that local extinctions are roughly matched by immigrations; that a high diversity of native species neither helps nor hinders the introduction of exotic species; and that exotic species find it much more difficult to invade native habitats than those modified by human activity. Of course, when the native species are endemic, the exotic species have come from elsewhere and global biodiversity is thereby reduced even if local avian biodiversity is maintained. We are moving towards an untextured world.

Case analyses three data sets for birds. The first is of 70 islands or archipelagos scattered around the world, plus two large continental land masses for which the number of native, established exotic and recently extinct native bird species can be estimated with reasonable accuracy. The number of native species is, as would be expected, well predicted by island area. But, although the number of extinct native species and estab- lished introductions are well correlated with each other (accounting for about $44 \%$ of the variance in numbers among the islands), they are uncorrelated with either island area or the number of native species. This finding does not accord with Charles Elton's suggestion that more speciose communities are more resistant to invasion by exotics ${ }^{3}$.

As conservation biologists are well aware, exotics can become successfully established only if a viable population is introduced into an area. To what degree, then, is the number of established exotic species dependent on the total number of exotic species that could have established populations (either by natural invasion or by human importation)? In his second data set, Case identifies a subset of 22 locations for which these data are available. Across locations, there is a positive correlation between the number of species that have successfully established populations and those that have failed, which accounts for about $30 \%$ of the variance across locations in the number of successfully established exotic species. However, when the number of failures is controlled for statistically, the number of successes remains strongly correlated with the number of native species that have become extinct.

Are the native extinctions and successful introductions a consequence of native habitats being destroyed and subsequently replaced by new habitats to which native

\title{
Exhibition \\ Heard the one about the guinea pig?
}

This striking view of the inside of a guinea-pig inner ear is just one of the prizewinning images that are on display as part of the Biomedical Image awards at the Wellcome Centre for Medical Science in London. Although the spiral cochlea is only a few millimetres long, the photograph - taken by David Furness of the Department of Neuroscience and Communication at Keele University using scanning electron microscopy - reveals rows of sensory cells which respond to different frequencies of sound, running along the length of the structure. The winning images were chosen from new material acquired by the Wellcome Centre Medical Photographic Library, and the exhibition runs until 25 April 1997. Alison Mitchell

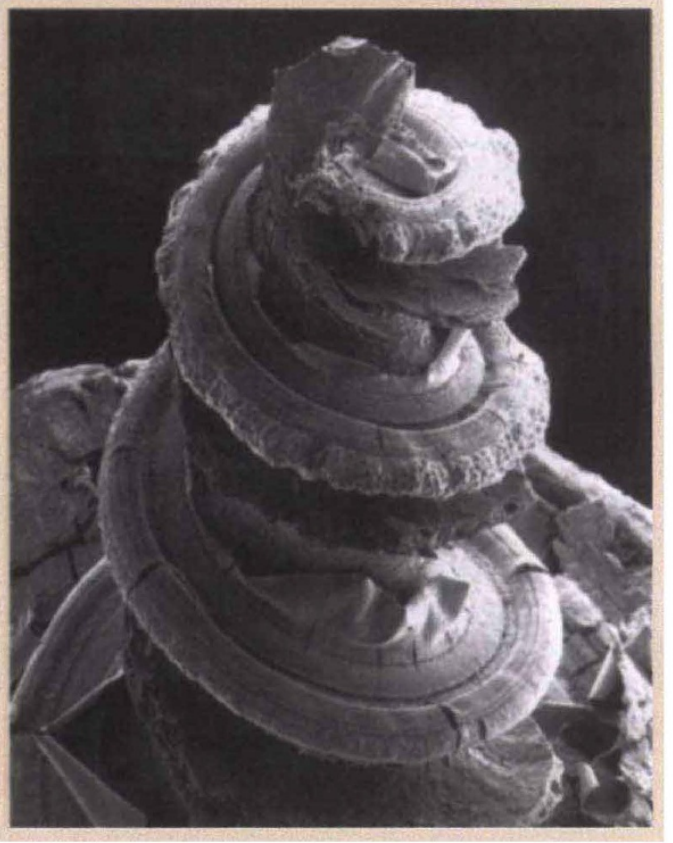

